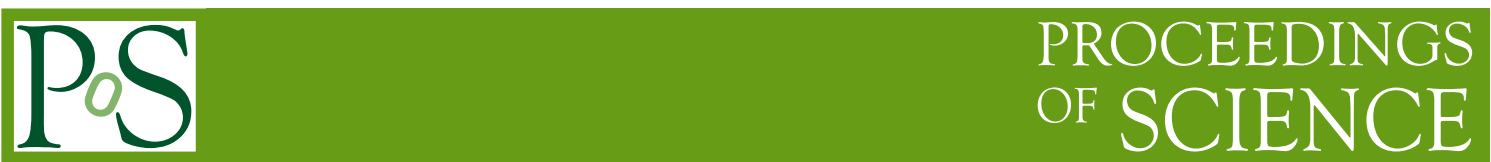

\title{
Hadroproduction measurements for simulations of neutrino beams
}

\section{MAURIZIO BONESINI*}

Sezione INFN Milano Bicocca, Dipartimento di Fisica G. Occhialini,

Piazza Scienza 3, Milano, Italy

E-mail: maurizio.bonesini@mib.infn.it

Recent $v$ experiments at accelerators have been complemented by ancillary hadron production experiments to provide additional informations on $\pi^{ \pm}, K^{ \pm}$cross sections, in the relevant phasespace regions, for a better understanding of beam systematics. The parametrization of these data is the base of fast simulation programs for neutrino beamlines, that may be very useful in their optimization phase. In addition, these data may be of interest for the tuning of general purpose MonteCarlo programs, such as FLUKA, MARS, GEANT4.

The European Physical Society Conference on High Energy Physics 18-24 July, 2013

Stockholm, Sweden

\footnotetext{
* Speaker.
} 
The prediction of the incoming $v$ flux and of its energy spectrum is an important element in accelerator experiments with conventional $v_{\mu}$ beams or superbeams [1]. The size of the minority components $\left(v_{e}, \overline{v_{e}}, \overline{v_{\mu}}\right)$ in a $v_{\mu}$ beam is difficult to evaluate and has relevant implications in neutrino oscillation experiments. Starting with the NA56/SPY experiment [2] for the NOMAD $v$ experiment at CERN WANF in the late 90-ties, modern accelerator $v$ experiments, such as MINOS, $\mathrm{K} 2 \mathrm{~K}$, MiniBooNE, T2K, ... have been complemented by ancillary hadron-production experiments as MIPP [3], HARP [4], NA61 [5] (see also table 1 for details) to provide additional informations on $\pi$ and $K$ production in the relevant phase space regions. In addition of taking data with a replica of the used $v$ production target, more general data on hadron production on thin targets, such as $\mathrm{Be}, \mathrm{C}, \mathrm{Al}, \ldots$, were taken. These data may be of interest also for the tuning of general purpose $\mathrm{MC}$, such as FLUKA, GEANT4 or MARS. .

Parametrizations of the available hadroproduction data are available at low-energy ( $p_{\text {inc }} \leq 30$ $\mathrm{GeV} / \mathrm{c}$ ), as the Sanford-Wang (SW) parametrization [6] and at high-energy, as the Atherton et al. [7], Malensek [8] and BMPT [9] parametrizations. One goal is to adopt a simple functional form appropriate for extrapolation to different center of mass energies and/or secondary particle momenta. In addition the possibility to extend the scope to different targets (e.g from Be to C) may be useful. These parametrization describe $\pi / K$ forward production and are used for the fast parametrizations of neutrino beams.
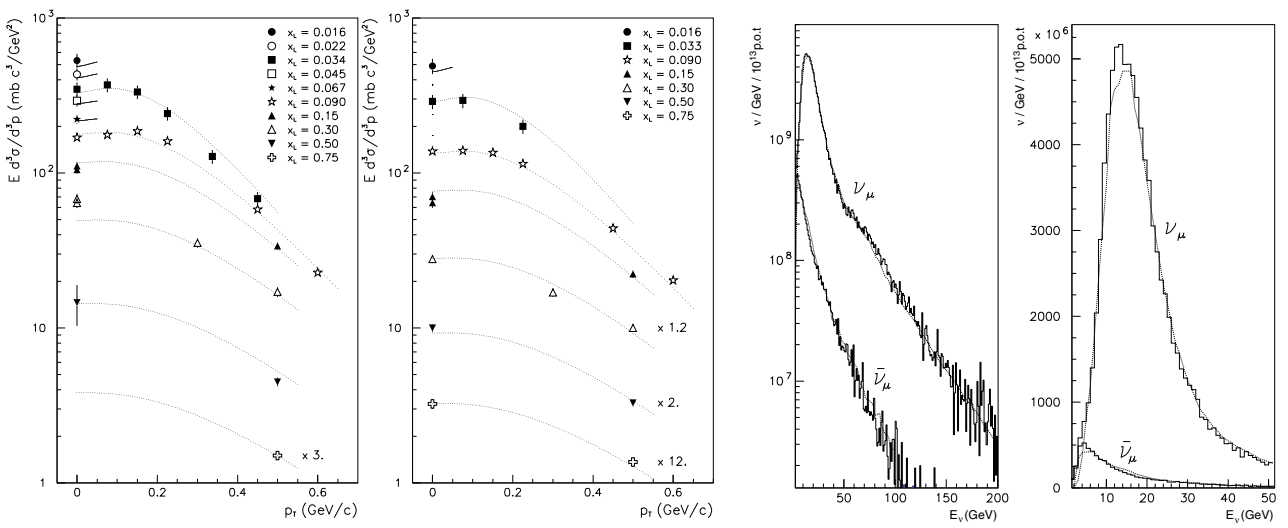

Figure 1: Left panel: BMPT parametrization, superimposed to NA56/SPY and NA20 data, for $\pi^{+}$(left) and $\pi^{-}$(right) in p-Be interactions. Right panel: the WANF neutrino fluxes at the CHARM2 detector: the dotted lines are the experimental data [12], the continuos line the fast beam simulation from reference [9].

Table 1: Dedicated hadron production experiments for $v$ beam studies. SAS (OG) means a single arm (open geometry) apparatus.

\begin{tabular}{|c|c|c|c|}
\hline Experiment & target & $p_{\text {beam }}(\mathrm{GeV} / \mathrm{c})$ & detector \\
\hline NA20 & $\mathrm{Be}$ & 400 & SAS \\
NA56/SPY & $\mathrm{Be}$ & 450 & SAS \\
HARP & $\mathrm{Be}, \mathrm{C}, \mathrm{Al}, \mathrm{Sn}, \mathrm{Ta}, \mathrm{Pb}, \mathrm{N}, \mathrm{O}, \mathrm{H}, \mathrm{H}_{2} \mathrm{O}$ & $1.5-15$ & OG \\
NA61/SHINE & $\mathrm{C}$ & 31 & OG \\
E907/MIPP & $\mathrm{LH}, \mathrm{Be}, \mathrm{C}, \mathrm{Al}, \mathrm{Bi}, \mathrm{U}$ & $5-80$ & OG \\
\hline
\end{tabular}

A comparison of the BMPT parameterization for $\pi^{ \pm}$production in $p-B e$ interactions [9], with 
available high energies data is shown in the left panel of figure 1. The proposed parametrization was found adequate to describe data from about $400 \mathrm{GeV} / \mathrm{c}$ down to $30 \mathrm{GeV} / \mathrm{c}$ with $10 \%$ accuracy. As an example, figure 2 reports the comparison of the original BMPT parametrization with 100
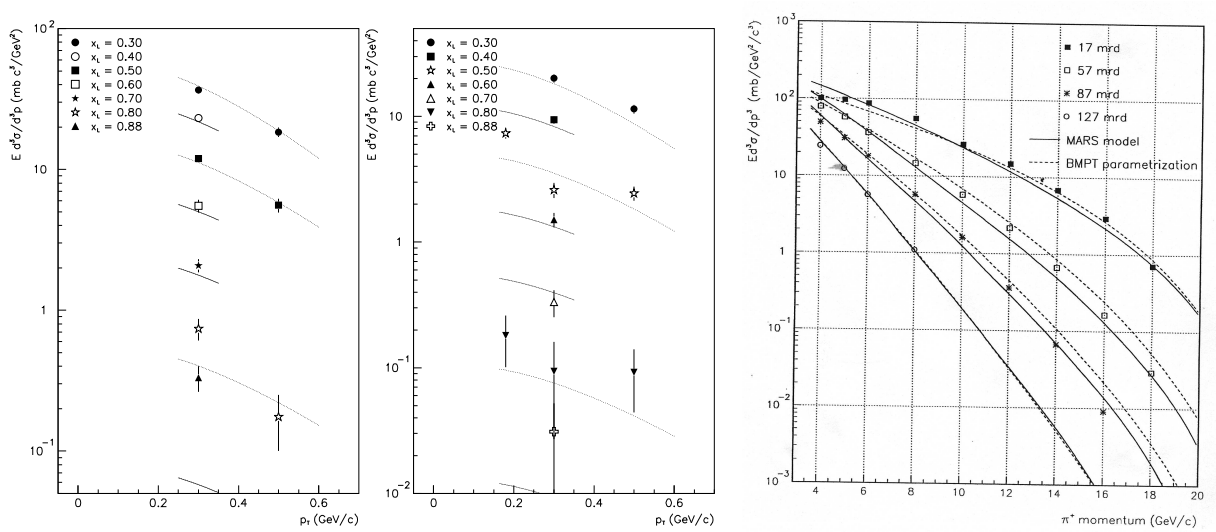

Figure 2: Left panel: comparison of the original BMPT parametrization with Barton et al. data [10] at 100 GeV/c. Right panel: comparison of the BMPT parametrization with T. Eichten et al. data [11].

$\mathrm{GeV} / \mathrm{c}$ data from reference [10] and $24 \mathrm{GeV} / \mathrm{c}$ data from reference [11]. Fast simulations based on such type of parametrizations may be of limited use for a full appreciation of neutrino beamline systematics, due to the approximations involved, but may be extremely useful in their optimization phase, where a fast program is needed. In order to give a more quantitative appreciation of the accuracy that one can obtain in fast simulations of neutrino beams, the right panel of figure 1 shows the comparison between the CHARM2 data [12] and the fast simulation from reference [9]. The overall agreement is at the percent level, with at most $10 \%$ disagreement on a bin per bin basis.

To improve agreement, the parameters of the BMPT parametrization were refitted to the NA61 $p-C$ data at $31 \mathrm{GeV} / \mathrm{c}$, before use in the beamline study for the T2K experiment at JPARC [13].

\section{References}

[1] M. Bonesini, A. Guglielmi Phys. Rept. 433 (2006) 65

[2] G. Ambrosini et al. [NA56/SPY Coll.], Eur. Jour. Phys. C10 (1999) 605.

[3] Y. Fysak et al. [MIPP Coll.], P-907 proposal, FNAL, 2000.

[4] M.G.Catanesi et al. [HARP Coll.], Nucl. Phys. B732 (2006) 1.

[5] M. Ungar et al. [NA61 Coll], arXiv:1305.5281

[6] J.R. Sanford, C.L. Wang BNL/AGS Internal Report JRS/CLW-1 (1967).

[7] H.W. Atherton et al. [NA20 Coll.], CERN 80-07, 1980.

[8] A.J. Malensek, FERMILAB FN-341 (1981)

[9] M. Bonesini, A. Marchionni, F. Pietropaolo, T. Tabarelli, Eur. Jour. Phys. C20 (2001) 13.

[10] D.S.Barton et al., Phys. ReV. D27 (1983) 2580.

[11] T.Eichten et al., Nucl. Phys. B44 (1972) 333.

[12] M. Jonker et al., Phys. Lett. 96B (1980) 435.

[13] K.Abe et al., Phys. ReV. D87,012001(2013) 